\title{
¿Cómo fomentar el desarrollo de competencias en la formación on-line? Una experiencia en la asignatura de dirección estratégica
}

\section{How to improve the competences develoment in the e-learning? A practical experience in the strategic management subject}

\section{Eva-María Mora-Valentín \\ Universidad Rey Juan Carlos, España \\ evamaria.mora@urjc.es \\ Fecha de recepción: 18-02-2014 \\ Fecha de revisión: 03-02-2015}

Palabras clave:

Competencias; Formación On-line; Dirección Estratégica; Universidad.

\section{Resumen}

El objetivo de este trabajo es analizar el desarrollo de competencias en la formación on-line a través de las actividades propuestas por el profesor en la guía docente. Para ello, se ha realizado un estudio exploratorio en la asignatura de Dirección Estratégica y Política de Empresa I que se imparte en modalidad on-line en el Grado de Administración y Dirección de Empresas en la Universidad Rey Juan Carlos. Nuestros resultados han puesto de manifiesto que existen diferencias entre la percepción del profesor y la del estudiante sobre las actividades que sirven para desarrollar cada una de las competencias definidas. Por ello, el docente debería reflexionar sobre cuáles son
Marta Ortiz-De-Urbina-Criado

Universidad Rey Juan Carlos, España marta.ortizdeurbina@urjc.es

Fecha de aceptación: 21-05-2015 Fecha de publicación: 15-06-2015

Keywords:

Competences; E-learning; Strategic Management; University.

\begin{abstract}
The aim of this paper is to analyze the development of competences in the on-line learning through the activities proposed by the teacher in the teaching guide. To do this, we have made an exploratory study on the on-line subject of Strategic Management and Business Policy I taught in the Degree of Business Administration at the Rey Juan Carlos University. Our results have shown that there are differences between the per-ception of the teacher and student on the activities used to develop the competences. Therefore, the teacher should reformulate the activities it proposes to achieve the competencies and to define clearly each competence and how it is acquired.
\end{abstract}


las actividades que propone para trabajar cada una de las competencias y, mucho más importante, definir bien qué entiende por cada competencia y cómo se alcanza.

\section{Introducción}

La implantación de los grados en el nuevo contexto educativo supone la necesidad de llevar a cabo ciertos cambios en la docencia universitaria con el objetivo de formar a los estudiantes en unas competencias prácticas que les capaciten para el ejercicio de su labor profesional. En este escenario, el diseño de los grados exige la definición clara de competencias y actividades que permitan al estudiante adquirir o desarrollar un nivel adecuado de dichas competencias.

La puesta en marcha de modelos educativos de gestión por competencias es un proceso bastante complejo (Guerrero-Roldán, Huertas, Mor, \& Rodríguez, 2013). Esta comple-jidad puede ser mayor en el caso de las universidades que ofrecen enseñanza on-line. Las características específicas de este tipo de formación hacen necesario definir claramente el tipo de actividades a desarrollar para que el estudiante pueda adquirir las competencias definidas en la guía docente de la asignatura. El uso de plataformas como WebCT o Moodle resulta fundamental en la puesta en práctica de esta formación virtual (Betegón Sánchez, Fossas Olalla, Martínez Rodríguez, \& Ramos González, 2010; Martín Galán \& Rodríguez Mateos, 2012), facilitando la generación de competencias por parte del estudiante.

Como consecuencia de ello, se ha producido un crecimiento importante en el número de trabajos que analizan diferentes aspectos de los procesos de adaptación de asignaturas de los planes antiguos a los nuevos o de la implantación de las asignaturas en los grados, entre los que destacan especialmente la implantación de modelos educativos por competencias tanto en formación presencial como on-line (Bustos Lambert, Arjona Fuentes, \& Celaya Pomposo, 2007; Ceular Villamandos, Núñez Tabales, Serrano $\mathrm{Mu}-$ ñoz, \& Caridad y Ocerin, 2012; GuerreroRoldán et al., 2013).

El objetivo de este trabajo es analizar el modelo de competencias en la asignatura de Dirección Estratégica y Política de Empresa I que se imparte a través de la plataforma Moodle en el Grado en Administración y Dirección de Empresas On-line de la Universidad Rey Juan Carlos. Para ello se han identificado las competencias que se pueden adquirir en la docencia on-line y se ha contrastado en qué medida cada una de las actividades realizadas permite al alumno el desarrollo de determinadas competencias. 


\section{Planteamientos teóricos}

\subsection{Formación por competencias}

El desarrollo de competencias se ha Manjón \& Pérez López, 2008).

constatado como uno de los pilares básicos En este contexto, el sistema universitario en la implantación de los estudios de grado europeo ha definido los grados en base a en el EEES (Espacio Europeo de Educación un conjunto de competencias generales y Superior). El concepto competencia está específicas que deben ser adquiridas por formado por tres dimensiones: el saber los estudiantes, siendo necesario alinear (disponer de los conocimientos técnicos cada competencia con las actividades que necesarios para la tarea), el saber hacer realiza el estudiante. Este modelo basado (capacidad de aplicar y utilizar dichos en las competencias deja de lado el logro de conocimientos mediante el despliegue de objetivos, para centrarse en las competencias las habilidades y destrezas apropiada) y el a alcanzar y el grado de adquisición de cada saber ser (adoptar las actitudes y desarrollar una de ellas, de forma que los estudiantes el comportamiento adecuado a las normas y son evaluados por competencias (procesos) cultura de la organización) (Abad Guerrero y no solo por contenidos (conocimientos). \& Castillo Clavero, 2004). Por tanto, las A su vez, los criterios de evaluación de competencias pueden definirse como un cada actividad deben reflejar el grado de conjunto de comportamientos observables que llevan a desempeñar eficaz y eficientemente un trabajo determinado en una organización concreta (Pereda, Berrocal, \& López, 2002).

En este trabajo distinguimos entre competencias genéricas y específicas. Las competencias genéricas se definen como una conducta asociada con el desarrollo, que es común a las distintas ocupaciones y ramas de actividad y que, a su vez, se pueden clasificar en instrumentales, interpersonales y sistémicas; las competencias específicas hacen referencia a un conjunto de conocimientos relevantes para cada tipo de trabajo (García adquisición de cada competencia. Tanto la calificación emitida como el feedback que recibe el estudiante deben estar relacionados con las competencias para poder mostrar al estudiante su progresión (Guerrero-Roldán et al., 2013). Este cambio de paradigma, supone un replanteamiento del proceso de evaluación para alinearlo con el logro de competencias (Avargil, Herscovitz, \& Dori, 2012).

Sin embargo, no debemos olvidar la complejidad que presenta el diseño e implantación de modelos educativos por competencias (Guerrero-Roldán et al., 2013). Esta complejidad resulta aún mayor en el caso 
de la enseñanza a distancia. La comunicación asíncrona y la no presencialidad de estos entornos de aprendizaje virtuales hacen necesario definir claramente cómo se trabajan y evalúan las habilidades y destrezas del estudiante. Para ello, es importante diseñar las actividades que deben realizarse para el desarrollo o adquisición de cada una de las competencias de la asignatura. Otro problema añadido es que si bien, la identificación de competencias está bastante bien definida

\subsection{Docencia on-line}

El concepto de educación a distancia incluye todas las formas de enseñanza en la que el alumno no acude de forma presencial a las clases como la teleformación, la enseñanzaaprendizaje virtual, la enseñanza on-line o el e-learning (García Aretio, 1986). Aguaded Gómez, Tirado Morueta y Hernando Gómez (2011) se refieren a la teleformación como una modalidad educativa apoyada por las tecnologías de la información y comunicación (TICs) que se desarrolla dentro del aprendizaje abierto y a distancia (open and distance learning). En este trabajo, nos centramos en la docencia on-line, que implica docencia a distancia mediante el empleo de TICs y espacios virtuales.

La docencia on-line se ha convertido en una práctica cada vez más habitual en las universidades presenciales, pudiendo los estudiantes elegir entre formación presencial o no presencial. Incluso existen algunas universidades totalmente virtuales como la Oberta de Catalunya (UOC) que desarrollan en el ámbito laboral, no es así en el ámbito educativo (Oliveros Martín-Varés, 2006). De ahí que algunos autores adviertan que la tarea de definir los planes de estudio en términos de competencias está siendo más compleja en el ámbito universitario que en otros como el de la formación profesional debido a que, en general, los estudios universitarios están más desligados del ámbito laboral (Guerrero Serón, 1999).

toda su docencia a través de Internet, considerándose su Campus Virtual como uno de los más avanzados y completos del panorama universitario a nivel internacional (Mondéjar, Mondéjar, \& Vargas, 2007). Un claro indicador de esta nueva tendencia formativa es el creciente número de universidades abiertas a nivel mundial (Laviña \& Mengual, 2008). En España, por ejemplo, se crea en 2006 la Universidad a Distancia de Madrid (UDIMA) y en 2009 la Universidad Internacional de La Rioja (UNIR).

Superadas las primeras etapas de la puesta en marcha de la formación on-line, el rápido desarrollo de las TICs y la Web 2.0 han abierto nuevas posibilidades pedagógicas para este tipo de enseñanza. Las metodologías de este tipo de enseñanza se basan en un diálogo didáctico mediado entre el profesor (institución) y el estudiante que, ubicado en espacio diferente al de aquel, aprende de forma independiente $\mathrm{y} / \mathrm{o}$ cooperativa (García Aretio, 2001). El uso de plataformas 
virtuales diseñadas con fines docentes como WebCT o Moodle resulta de gran ayuda en esta modalidad de formación (Betegón Sán-chez et al., 2010; García Aretio \& Ruiz Corbella, 2010; Martín Galán \& Rodríguez Mateos, 2012). En la misma línea, Pavón Rabasco y Casanova Correa (2007) definen el aula virtual como un entorno educativo que intenta facilitar el aprendizaje cooperativo y colaborativo entre estudiantes, y entre estos y los profesores.

Existen muchos trabajos que han puesto de manifiesto las características específicas de la formación on-line. Siguiendo a Mondéjar et al., (2007), el e-learning es capaz de suprimir el contacto presencial por el contacto virtual, puede estar sujeto a una serie de normas o reglas de funcionamiento fijadas por los administradores del mismo, se puede controlar la cantidad de información suministrada en todo momento y, por último, el tamaño de los grupos (que era una variable prioritaria en la enseñanza presencial), pasa a un segundo plano, y solo será relevante para el hecho de no poder realizar un seguimiento por parte del profesor de todos sus alumnos. Para González Díaz-Caneja, López García y Chasco Irigoyen (2003), la formación online permite asistir a clase de forma remota así como crear un espacio virtual de reunión para la realización de trabajos, facilitando un aprendizaje más dinámico y una importante reducción de costes. En la misma línea, Acosta Lugo (2004) cita otras ventajas como la eliminación de la distancia física, la posibilidad de trabajo individual o en grupo,

el horario flexible, que está dirigida a un número mayor de destinatarios, el ritmo de aprendizaje individualizado, la participación de multiplicidad de expertos y la evaluación on-line. En definitiva, en la actualidad y debido a sus innumerables ventajas, la educación a distancia ha ido ganando terreno de forma progresiva a la modalidad presencial debido a que permite aprovechar al máximo el potencial educativo de la conectividad, de la relación asíncrona entre estudiantes y profesores, de forma que cuanto más tiempo se esté debatiendo, colaborando, compartiendo conocimiento $\mathrm{y}$, en definitiva, aprendiendo, mejores resultados académicos se obtendrán (Duart, 2010).

Sin embargo, la formación on-line también presenta algunos problemas como las elevadas tasas de abandono o fracaso que, con frecuencia, son más altas que en los formatos presenciales. Una de las explicaciones de esta alta tasa de fracaso se encuentra en el sentimiento de soledad del estudiante, especialmente al inicio del periodo formativo. Se trata de un alumno que físicamente se encuentra solo ante unos materiales y recursos, en un entorno muchas veces poco favorecedor para el estudio. Por eso, las estrategias necesarias para el estudio on-line difieren de las presenciales, y el grado de autonomía del estudiante es sensiblemente mayor desde el inicio de su proceso de aprendizaje, lo que conlleva la adquisición de unas destrezas específicas (García Aretio \& Ruiz Corbella, 2010). 


\subsection{Desarrollo y adquisición de competencias en la docencia on-line}

El proceso de transformación de los planes antiguos en grados ha supuesto un cambio importante en la regulación de las asignaturas, sustituyéndose el programa de la asignatura por la guía docente. Siguiendo a García Martín (2010), la guía docente constituye una planificación detallada de cualquier asignatura basada en los principios que guían el proceso de convergencia en la creación del EEES. Si en los antiguos programas el eje se situaba sobre el contenido (selección de contenidos, estructura y distribución en el programa, criterios para su evaluación, etc.) en este caso el eje se centra tanto en las competencias propias de la asignatura como en el trabajo del estudiante para adquirir esas competencias. Constituye un documento en el que se especifican todos los aspectos relevantes de una asignatura, esto es, los objetivos formativos, las competencias que se adquieren, el programa, la metodología, la bibliografía y el catálogo de técnicas docentes, de actividades académicas y de métodos de evaluación.

Para el profesor, la elaboración de la guía docente supone un auténtico ejercicio de planificación y reflexión, para relacionar los objetivos del aprendizaje con las competencias que se pretenda desarrollar con la asignatura, a través de las actividades formativas y empleando la evaluación como elemento de seguimiento y control del aprendizaje. Sin embargo, uno de los problemas fundamentales que se ha puesto de manifiesto con las guías docentes es que si bien la metodología empleada permite al alumno la adquisición de conocimientos teóricos, no suele contemplar la forma en que el estudiante puede desarrollar las competencias que en ella se definen, es decir, no se definen actividades que favorezcan la adquisición de dichas competencias.

Por otro lado y debido a las diferencias que existen entre la formación presencial y la on-line, la aplicación directa de metodologías formativas presenciales en escenarios de educación on-line hace que los niveles de calidad formativos se resientan considerablemente. En la docencia on-line, el diseño de la asignatura debe contribuir a que el alumno pueda asimilar los conocimientos que se le transmiten así como desarrollar las competencias definidas en la guía. En este sentido, el papel desarrollado por el profesor resulta fundamental al tener que identificar cuáles son las competencias que se pueden generar con la docencia on-line y definir el tipo de actividad que debe realizar el alumno para adquirir cada competencia. 


\section{Metodología}

\subsection{Experiencia práctica}

Para tener una perspectiva general de las que más relación tienen con las titulaciones competencias en los estudios de Dirección de de Empresa y, más concretamente con la Empresas se han analizado las conclusiones disciplina de Dirección de Empresas ${ }^{1}$.

del Tuning Education Structures in Europe En este trabajo nos centramos en la (González \& Wagenaar, 2003) así como las asignatura de Dirección Estratégica y Política del Libro Blanco del Título en Economía de Empresa $I^{2}$, que se imparte a través de y Empresa (ANECA, 2005). Ambos la plataforma Moodle en $3^{\mathrm{o}}$ del Grado en documentos, el primero a nivel europeo y el Administración y Dirección de Empresas Onsegundo a nivel nacional (España), muestran line de la Universidad Rey Juan Carlos, en tanto las competencias genéricas que deben el curso 2013/14. Esta asignatura tiene una conseguir los estudiantes universitarios, carga docente de 6 créditos, se imparte en como las específicas propias de cada área de el primer cuatrimestre, e incluye todos los conocimiento. Partiendo de estos trabajos, en temas relativos al análisis estratégico y la la Tabla 1, hemos recogido las competencias formulación de las estrategias competitivas.

\begin{tabular}{|c|c|c|}
\hline \multirow{13}{*}{ Genéricas } & \multirow{8}{*}{$\begin{array}{c}\text { Instrumentales } \\
\text { (8) }\end{array}$} & Capacidad de análisis y síntesis \\
\hline & & Capacidad de organización y planificación \\
\hline & & Comunicación oral y escrita en lengua nativa \\
\hline & & Comunicación oral y escrita de una lengua extranjera \\
\hline & & $\begin{array}{l}\text { Conocimientos de informática relativos al ámbito de estu- } \\
\text { dio }\end{array}$ \\
\hline & & $\begin{array}{l}\text { Habilidad para analizar información proveniente de fuentes } \\
\text { diversas }\end{array}$ \\
\hline & & Capacidad para la resolución de problemas \\
\hline & & Capacidad para tomar decisiones \\
\hline & \multirow{5}{*}{$\begin{array}{l}\text { Personales } \\
\text { (8) }\end{array}$} & Capacidad para trabajar en equipo \\
\hline & & Trabajo en un equipo de carácter interdisciplinar \\
\hline & & Trabajo en un contexto internacional \\
\hline & & Habilidad en las relaciones personales \\
\hline & & $\begin{array}{l}\text { Capacidad para trabajar en entornos diversos y multicultu- } \\
\text { rales }\end{array}$ \\
\hline
\end{tabular}




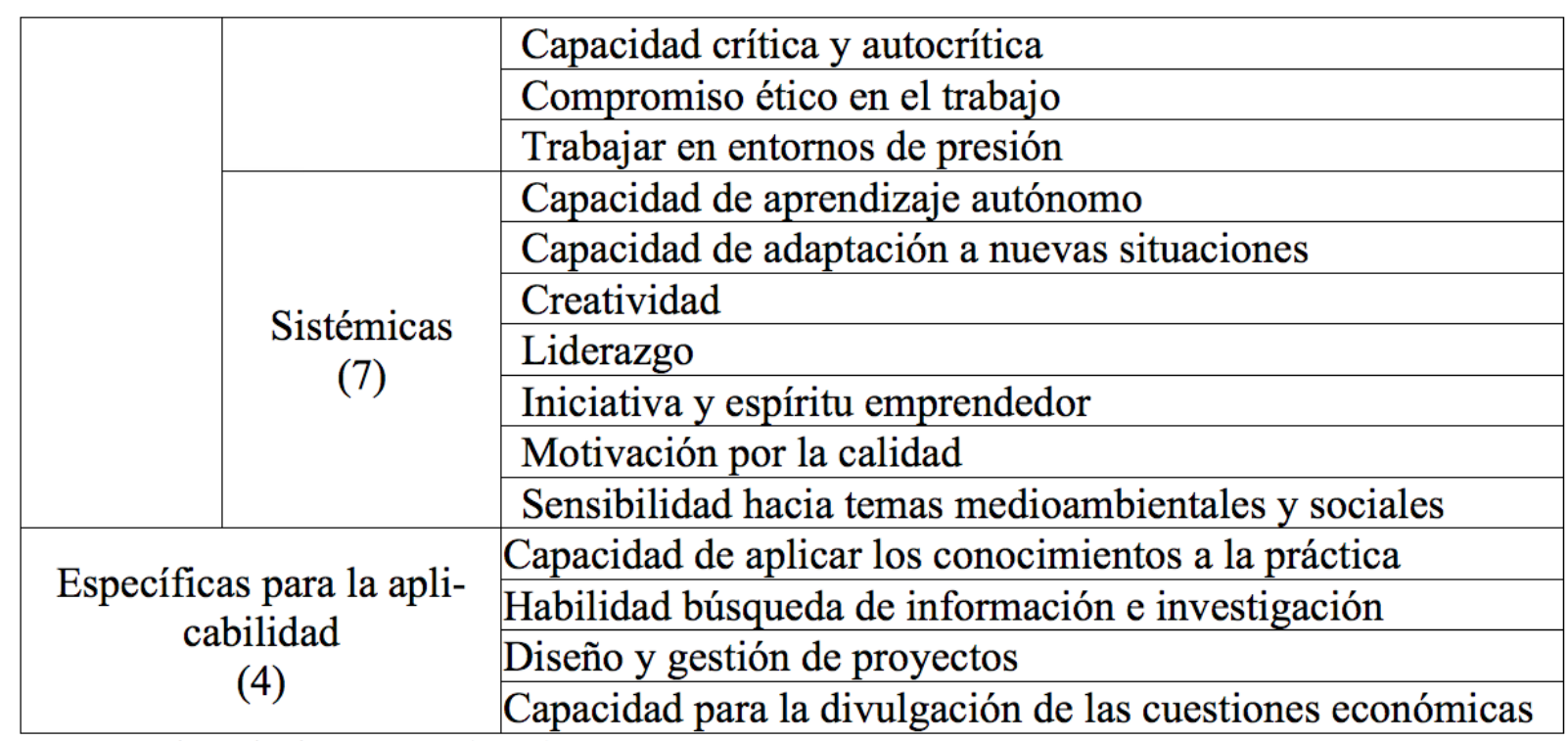

Fuente: adaptado de ANECA (2005).

Tabla 1. Competencias genéricas y específicas en Dirección de Empresas.

Teniendo en cuenta que la asignatura se imparte on-line, hemos procedido a identificar solamente aquellas competencias que mejor se adaptan a esta modalidad formativa (Tabla 2). En primer lugar, de las ocho competencias instrumentales hemos seleccionado seis debido a dos motivos. Teniendo en cuenta que la asignatura se imparte en castellano, no se ha incluido la competencia comunicación oral y escrita de una lengua extranjera; por otro lado, también hemos eliminado la competencia conocimientos de informática relativos al ámbito de estudio ya que en el temario no se incluyen aspectos relacionados la informática. En segundo lugar, no se han considerado cuatro de las ocho competencias personales: trabajo en un equipo de carácter interdisciplinar, trabajo en un contexto internacional, habilidad en las relaciones personales y capacidad para trabajar en entornos diversos y multiculturales. Ello se debe a una de las principales limitaciones de la formación on-line: la inexistencia del trato cara a cara profesor-alumno. En tercer lugar, hemos considerado todas las competencias sistémicas excepto la relativa a temas medioambientales. Por último y respecto a las competencias específicas, hemos excluido las relacionadas con el diseño y gestión de proyectos y con la capacidad para la divulgación de las cuestiones económicas, por ser cuestiones no tratadas en la asignatura.

La asignatura incluye un total de nueve temas que se evalúan en un $30 \%$ con las prácticas realizadas y en un $70 \%$ con un examen final. Para la parte práctica se han propuesto seis actividades obligatorias y evaluables con las que se pretende que el alumno desarrolle un conjunto de competencias que han sido identificadas a criterio del docente. Para ello y con el objetivo de analizar la relación competencia-actividad, se llevó a cabo un minucioso proceso que se materializó en un listado de varias competencias vinculadas a cada una de las actividades propuestas (Tabla 3). 


\begin{tabular}{|c|c|c|c|}
\hline \multicolumn{3}{|r|}{ Competencias } & Código \\
\hline \multirow{16}{*}{ Genéricas } & \multirow{6}{*}{$\mid \begin{array}{c}\text { Instrumentales } \\
(6)\end{array}$} & Capacidad de análisis y síntesis & CAS \\
\hline & & Capacidad de organización y planificación & COP \\
\hline & & Comunicación escrita en lengua nativa & CEN \\
\hline & & $\begin{array}{l}\text { Habilidad para analizar información prove- } \\
\text { niente de fuentes diversas }\end{array}$ & CAIF \\
\hline & & Capacidad para la resolución de problemas & CRP \\
\hline & & Capacidad para tomar decisiones & CTD \\
\hline & \multirow{4}{*}{$\begin{array}{l}\text { Personales } \\
\text { (4) }\end{array}$} & Capacidad para trabajar en equipo & CTE \\
\hline & & Capacidad crítica y autocrítica & $\mathrm{CCA}$ \\
\hline & & \begin{tabular}{|l|} 
Compromiso ético en el trabajo \\
\end{tabular} & CET \\
\hline & & Trabajar en entornos de presión & TEP \\
\hline & \multirow{6}{*}{$\begin{array}{l}\text { Sistémicas } \\
\quad(6)\end{array}$} & Capacidad de aprendizaje autónomo & CAA \\
\hline & & $\begin{array}{l}\text { Capacidad de adaptación a nuevas situacio- } \\
\text { nes }\end{array}$ & CANS \\
\hline & & Creatividad & CRE \\
\hline & & Liderazgo & LID \\
\hline & & Iniciativa y espíritu emprendedor & IEE \\
\hline & & Motivación por la calidad & MPC \\
\hline \multirow{2}{*}{\multicolumn{2}{|c|}{$\begin{array}{l}\text { Específicas para la apli- } \\
\text { cabilidad } \\
(2)\end{array}$}} & $\begin{array}{l}\text { Capacidad de aplicar los conocimientos a la } \\
\text { práctica }\end{array}$ & ССР \\
\hline & & $\begin{array}{l}\text { Habilidad búsqueda de información e investi- } \\
\text { gación }\end{array}$ & HBI \\
\hline
\end{tabular}

Fuente: adaptado de ANECA (2005).

Tabla 2. Competencias genéricas y específicas en la asignatura Dirección Estratégica y Política de Empresa I.

Teniendo en cuenta las actividades realizadas la segunda parte, el alumno debe valorar y la selección de competencias, para me- en qué medida cada una de las actividades dir el grado de adquisición de competencias realizadas le ha permitido desarrollar cada por parte de los estudiantes, al final del una de las competencias seleccionadas. Todos cuatrimestre (diciembre de 2013) se realizó los alumnos que realizaron las prácticas a un cuestionario on-line (a través de Moodle) lo largo del cuatrimestre cumplimentaron que consta de dos partes. En la primera el cuestionario, es decir, un total de treinta parte, se pide al alumno que valore en qué y siete alumnos de los cuarenta y tres grado tenía disponible cada competencia matriculados en la asignatura $(86 \%)$. antes y después de cursar la asignatura. En

\subsection{Resultados}

A partir de la información obtenida a través de este cuestionario se han realizado varios análisis estadísticos con diferentes objetivos. En primer lugar, para evaluar las diferencias que hay entre el grado de competencia que tenía el alumno antes y después de cursar la asignatura. En segundo lugar, para valorar en qué medida cada una de las actividades 


\begin{tabular}{|c|c|l|c|}
\hline Temas & $\begin{array}{c}\text { N}^{0} \text { acti- } \\
\text { vidad }\end{array}$ & \multicolumn{1}{|c|}{ Actividad } & $\begin{array}{c}\text { Competencias a } \\
\text { desarrollar }\end{array}$ \\
\hline 1 & 1 & Estudio dirigido & $\begin{array}{c}\text { CAS; COP; CEN; } \\
\text { CAA }\end{array}$ \\
\hline 2 y 3 & 2 & $\begin{array}{l}\text { Análisis de la creación de valor, gru- } \\
\text { pos de interés, objetivos y valores de } \\
\text { una empresa }\end{array}$ & $\begin{array}{c}\text { CAS; COP; CEN; } \\
\text { CAIF; CCP; HBI }\end{array}$ \\
\hline 4 & 3 & $\begin{array}{l}\text { Foro para identificar los factores del } \\
\text { entorno general de una empresa }\end{array}$ & $\begin{array}{c}\text { CAS; CEN; CAIF; } \\
\text { CTD; CRE; CCP; HBI }\end{array}$ \\
\hline 5 & 4 & $\begin{array}{l}\text { Aplicar el modelo de las 5 fuerzas } \\
\text { competitivas de Porter a un sector }\end{array}$ & $\begin{array}{c}\text { CAS; COP; CAIF; } \\
\text { CCP; HBI }\end{array}$ \\
\hline 6 & 5 & $\begin{array}{l}\text { Realizar el análisis DAFO de una em- } \\
\text { presa }\end{array}$ & $\begin{array}{c}\text { CAS; COP; CAIF; } \\
\text { CCA; CET; CCP; HBI }\end{array}$ \\
\hline 8 & 6 & $\begin{array}{l}\text { Caso práctico en grupo sobre la venta- } \\
\text { ja competitiva de una empresa }\end{array}$ & $\begin{array}{c}\text { CAS; COP; CEN; } \\
\text { CAIF; CRP; CTE; } \\
\text { CRE; CCP; HBI }\end{array}$ \\
\hline
\end{tabular}

Tabla 3. Asignación de actividades a competencias a criterio del profesor.

realizadas le ha permitido desarrollar cada una de las competencias seleccionadas y analizar si se corresponden con las competencias que el profesor quería desarrollar al proponer cada una de las actividades. Para el logro del primer objetivo, se ha realizado un análisis de frecuencias $^{3}$ y de diferencias entre las competencias disponibles antes y después de cursar la asignatura (Tabla 4).

Para las competencias genéricas instrumentales, se obtienen valores medios de entre 6,81 y 7,54 antes de cursar la asignatura, mientras que después de hacerlo la media sube y oscila entre 8,08 y 8,32 . Para las competencias genéricas personales se obtienen valores medios de entre 7,03 y 7,81 antes de cursar la asignatura y de entre 7,92 y 8,43, después de hacerlo. También para las competencias genéricas sistémicas y para las específicas para la aplicabilidad se obtienen resultados similares. Para las primeras, los valores medios oscilan entre 6,59 y 7,65 antes de cursar la asignatura y entre
7,46 y 8,46 después de hacerlo. En el caso de las competencias específicas, se observa un aumento mayor en los valores medios, oscilando entre 6,84 y 7,14 antes de cursar la asignatura y entre 8,32 y 8,43 después de hacerlo.

Si analizamos las correlaciones entre el grado en que el alumno tenía disponible cada competencia antes y después de cursar la asignatura, observamos que entre muchas de las competencias hay correlación media o alta (superior a 0,4). Sin embargo hay algunos casos en los que la correlación es baja; esto ocurre para la capacidad para tomar decisiones, el compromiso ético en el trabajo y la capacidad para aplicar los conocimientos a la práctica. Por otra parte, la Tabla 4 muestra que para diecisiete de las dieciocho competencias analizadas hay diferencias significativas, es decir, el alumno considera que ha mejorado el grado en el que tenía disponible esa competencia una vez que ha cursado la asignatura. El único caso en 


\begin{tabular}{|c|c|c|c|c|c|c|}
\hline \multicolumn{2}{|c|}{ Competencias } & Correlación & Sig. & $\begin{array}{c}\text { Media (dife- } \\
\text { rencias) }\end{array}$ & t $*$ & $\begin{array}{c}\text { Sig. (bila- } \\
\text { teral) }\end{array}$ \\
\hline \multirow{4}{*}{ CGI } & CAS & 0,57 & 0,000 & $-1,32$ & $-8,809$ & 0,000 \\
\cline { 2 - 7 } & COP & 0,72 & 0,000 & $-0,95$ & $-7,379$ & 0,000 \\
\cline { 2 - 7 } & CEN & 0,72 & 0,000 & $-0,62$ & $-4,986$ & 0,000 \\
\cline { 2 - 7 } & CAIF & 0,61 & 0,000 & $-1,11$ & $-7,703$ & 0,000 \\
\cline { 2 - 7 } & CRP & 0,66 & 0,000 & $-0,95$ & $-7,379$ & 0,000 \\
\cline { 2 - 7 } & CTD & 0,39 & 0,017 & $-0,70$ & $-3,712$ & 0,001 \\
\hline \multirow{4}{*}{ CGP } & CTE & 0,41 & 0,013 & $-0,70$ & $-3,792$ & 0,001 \\
\cline { 2 - 7 } & CCA & 0,54 & 0,000 & $-0,89$ & $-6,200$ & 0,000 \\
\cline { 2 - 7 } & CET & 0,35 & 0,036 & $-0,27$ & $-1,260$ & 0,216 \\
\cline { 2 - 7 } & TEP & 0,66 & 0,000 & $-0,78$ & $-5,036$ & 0,000 \\
\hline \multirow{7}{*}{ CAA } & 0,77 & 0,000 & $-0,81$ & $-6,082$ & 0,000 \\
\cline { 2 - 7 } & CANS & 0,67 & 0,000 & $-0,81$ & $-5,258$ & 0,000 \\
\cline { 2 - 7 } & CRE & 0,85 & 0,000 & $-0,86$ & $-5,931$ & 0,000 \\
\cline { 2 - 7 } & LID & 0,69 & 0,000 & $-0,86$ & $-5,388$ & 0,000 \\
\cline { 2 - 7 } & IEE & 0,74 & 0,000 & $-0,95$ & $-5,763$ & 0,000 \\
\cline { 2 - 7 } & MPC & 0,71 & 0,000 & $-0,95$ & $-6,785$ & 0,000 \\
\hline \multirow{2}{*}{ CEA } & CCP & 0,39 & 0,019 & $-1,30$ & $-7,329$ & 0,000 \\
\cline { 2 - 7 } & HBI & 0,60 & 0,000 & $-1,49$ & $-10,394$ & 0,000 \\
\hline
\end{tabular}

* Prueba t de student para muestras relacionadas.

Tabla 4. Relación entre competencias antes y después de cursar la asignatura.

que el alumno no ha percibido una mejora significativa ha sido en el compromiso ético en el trabajo.

En segundo lugar, se ha realizado un análisis de en qué medida cada una de las actividades realizadas ha permitido al alumno desarrollar cada una de las competencias seleccionadas (Tabla 5).

En la actividad 1, se propuso un estudio dirigido del tema 1 con el objetivo de que el alumno fuera capaz de realizar un resumen del mismo que le sirviera como guía para el estudio de los contenidos teóricos. Con esta actividad se pretendía que el estudiante desarrollase cuatro competencias: capacidad de análisis y síntesis, capacidad de organización y planificación, comunicación escrita en lengua nativa y capacidad de aprendizaje autónomo (Tabla 3). Los alumnos consideraron que las competencias que se podían desarrollar en mayor medida (media superior a 8) eran la capacidad de análisis y síntesis y la capacidad de aprendizaje autónomo. También se obtienen valores medios altos (entre 7,5 y 8) para otras cuatro competencias: capacidad de organización y planificación, comunicación escrita en lengua nativa, motivación por la calidad y habilidad para la búsqueda de información e investigación (Tabla 5). Destacar que las dos últimas no fueron contempladas por el profesor ya que para realizar esta actividad solo se necesitaba el tema 1 del libro (y no otras fuentes adicionales) valorando solamente la capacidad de síntesis, de organización y de comunicación escrita. 


\begin{tabular}{|c|c|c|c|c|c|c|c|c|c|c|c|c|}
\cline { 2 - 16 } \multicolumn{1}{c|}{} & \multicolumn{2}{|c|}{ Actividad 1 } & \multicolumn{2}{c|}{ Actividad 2 } & \multicolumn{2}{c|}{ Actividad 3 } & \multicolumn{2}{c|}{ Actividad 4 } & \multicolumn{2}{|c|}{ Actividad 5 } & \multicolumn{2}{c|}{ Actividad 6 } \\
\hline & Media & $\begin{array}{c}\text { Desv. } \\
\text { típ. }\end{array}$ & Media & $\begin{array}{c}\text { Desv. } \\
\text { típ. }\end{array}$ & Media & $\begin{array}{c}\text { Desv. } \\
\text { típ. }\end{array}$ & Media & $\begin{array}{c}\text { Desv. } \\
\text { típ. }\end{array}$ & Media & $\begin{array}{c}\text { Desv. } \\
\text { típ. }\end{array}$ & Media & $\begin{array}{c}\text { Desv. } \\
\text { típ. }\end{array}$ \\
\hline CAS & 8,30 & 1,127 & 8,14 & 1,084 & 7,68 & 1,872 & 8,16 & 1,385 & 7,84 & 1,993 & 8,03 & 1,590 \\
\hline COP & 7,95 & 1,177 & 8,03 & 1,040 & 7,27 & 1,851 & 8,14 & 1,182 & 7,62 & 1,977 & 8,16 & 1,724 \\
\hline CEN & 7,78 & 1,315 & 8,14 & 1,004 & 7,27 & 2,064 & 7,89 & 1,286 & 7,22 & 1,873 & 7,97 & 1,536 \\
\hline CAIF & 7,38 & 1,605 & 8,54 & 1,043 & 7,78 & 1,946 & 8,27 & 1,347 & 7,89 & 1,969 & 8,30 & 1,631 \\
\hline CRP & 6,95 & 1,682 & 7,65 & 1,654 & 7,16 & 2,021 & 7,86 & 1,398 & 7,22 & 1,652 & 7,89 & 1,612 \\
\hline CTD & 6,95 & 1,699 & 7,76 & 1,321 & 7,32 & 1,886 & 7,89 & 1,410 & 7,22 & 1,734 & 8,19 & 1,596 \\
\hline CTE & 5,43 & 2,930 & 5,65 & 2,927 & 6,43 & 2,512 & 5,59 & 2,783 & 5,49 & 2,755 & 8,46 & 1,952 \\
\hline CCA & 6,81 & 2,145 & 7,65 & 1,136 & 7,54 & 1,693 & 7,86 & 1,228 & 7,27 & 2,023 & 8,22 & 1,584 \\
\hline CET & 7,30 & 1,793 & 7,95 & 1,129 & 7,46 & 1,820 & 7,62 & 1,861 & 6,86 & 2,647 & 8,08 & 1,754 \\
\hline TEP & 7,03 & 2,061 & 7,22 & 1,873 & 7,00 & 1,732 & 7,46 & 1,742 & 6,84 & 2,128 & 7,89 & 1,646 \\
\hline CAA & 8,27 & 1,097 & 7,95 & 1,490 & 7,89 & 1,629 & 8,30 & 1,102 & 7,76 & 2,006 & 7,92 & 1,754 \\
\hline CANS & 7,30 & 1,488 & 7,54 & 1,095 & 7,35 & 1,687 & 7,68 & 1,396 & 7,19 & 2,025 & 7,78 & 1,782 \\
\hline CRE & 6,92 & 1,460 & 7,32 & 1,600 & 7,03 & 1,787 & 7,62 & 1,233 & 6,86 & 2,084 & 7,46 & 1,773 \\
\hline LID & 6,11 & 2,470 & 6,76 & 2,178 & 6,51 & 1,850 & 6,78 & 1,734 & 6,16 & 2,267 & 7,89 & 1,776 \\
\hline IEE & 6,68 & 2,109 & 7,49 & 1,170 & 6,89 & 1,897 & 7,43 & 1,405 & 6,92 & 1,963 & 8,08 & 1,588 \\
\hline MPC & 7,95 & 1,353 & 8,03 & 1,280 & 7,54 & 1,709 & 8,16 & 1,167 & 7,51 & 1,909 & 8,19 & 1,647 \\
\hline CCP & 7,30 & 1,561 & 8,22 & 1,228 & 7,97 & 1,641 & 8,38 & 1,187 & 7,95 & 1,914 & 8,30 & 1,596 \\
\hline HBI & 7,54 & 1,406 & 8,46 & 1,070 & 8,03 & 1,756 & 8,32 & 1,375 & 7,65 & 2,071 & 8,30 & 1,561 \\
\hline
\end{tabular}

Tabla 5. Competencias y actividades.

El objetivo de la actividad 2 era buscar un ejemplo de una empresa real que pusiese de manifiesto algunos de los aspectos analizado en los temas 2 y 3 . El profesor seleccionó una empresa española y pidió a los alumnos que analizaran la creación de valor, los grupos de interés y los objetivos y valores de la misma. En este caso, la actividad se propuso para desarrollar o mejorar las siguientes competencias: CAS, COP, CEN, CAIF, CCP y HBI (Tabla 3). Los resultados (Tabla 5) muestran que los alumnos otorgaron los valores más altos a estas competencias (media superior a 8), pero también añadían una competencia más, la motivación por la calidad.

En la actividad 3 el alumno tenía que participar en un foro para identificar factores del entorno general para la empresa seleccionada por el profesor en la actividad 2 .
Cada alumno tenía que proponer un factor, clasificarlo y aportar información adicional para justificarlo (noticias de prensa, webs, libros, etc.). Esta actividad iba dirigida principalmente al desarrollo de varias competencias: CAS, CEN, CAIF, CTD, CRE, CCP y HBI (Tabla 3). La Tabla 5 muestra que la competencia más destacable es la HBI (media superior a 8) seguida por otras seis (media entre 7,5-7,9): CAS, CAIF, CCA, CAA, MPC y CCP. En este caso hay diferencias entre las competencias propuestas por el profesor y las valoradas por el alumno. Así, el alumno destaca algunas competencias no consideradas por el profesor, en concreto, capacidad de crítica y autocrítica, capacidad para el aprendizaje autónomo y motivación por la calidad, dando menos importancia a algunas de las propuestas por el profesor: comunicación escrita en lengua nativa y 
capacidad para tomar decisiones y creatividad. En la actividad 4 se pedía al alumno que, tras la lectura de un artículo sobre un sector, identificara las 5 fuerzas competitivas de Porter y justificara su efecto sobre el atractivo de la industria. En este caso el profesor destacó las siguientes competencias: CAS, COP, CAIF, CCP y HBI (Tabla 3). Los alumnos dieron valores altos a todas estas competencias (media superior a 8) pero también incluyeron dos más: capacidad de aprendizaje autónomo y motivación por la calidad (Tabla 5).

En la actividad 5 se pedía al alumno que hiciera un DAFO para la empresa seleccionada por el profesor para las actividades anteriores. Se indicaba al alumno que utilizase la información disponible tras la realización de la actividad 3 (foro). Las competencias que se planteaban para esta actividad eran: CAS, COP, CAIF, CCA, CET, CCP y HBI (Tabla 3). En este caso y tras comprobar la disponibilidad en Internet de algunos análisis DAFO, en muchos casos incompletos o incluso mal planteados, el profesor decidió incluir esta actividad para comprobar si el alumno tenía capacidad de crítica, un comportamiento ético y era capaz de utilizar y citar información proveniente de varias fuentes para realizar la actividad. Tras la realización de la actividad el profesor comprobó que muchos alumnos copiaron alguno de los análisis DAFO disponibles en la web, no incluyeron información proveniente de otras fuentes y no citaron la fuente de información utilizada. Los resultados muestran que algunas de las competencias propuestas por el profesor no fueron valoradas como las más importantes por los alumnos (medias inferiores a 7,5), en concreto, capacidad de crítica y autocrítica y comportamiento ético en el trabajo. Sin embargo, valoraron otras competencias como la capacidad de aprendizaje autónomo y la motivación por la calidad (media superior a $7,5)$.

La última actividad fue la única que se realizó en grupo. El profesor creó grupos de cuatro personas de forma aleatoria y les propuso la resolución de un caso práctico sobre las estrategias competitivas. Para ello, cada grupo tenía a su disposición herramientas como los foros, el chat y el correo. En esta actividad una de las principales competencias que se deseaba potenciar era la del trabajo en equipo (CTE). También se incluyeron otras como CAS, COP, CEN, CAIF, CRP, CRE, CCP y HBI (Tabla 3). Los resultados (Tabla 5) muestran que esta actividad es en la que se destaca un mayor número de competencias (media superior a 8). Tres de las competencias propuestas por el profesor no obtuvieron las puntuaciones más altas: CEN, CRP, CRE; sin embargo los alumnos valoraron otras como la capacidad para tomar decisiones, la capacidad de crítica y autocrítica, el compromiso ético en el trabajo, la iniciativa y espíritu emprendedor y la motivación por la calidad. Un aspecto destacable en este caso es la importancia que se da al compromi-so ético en el trabajo. Tras la realización de la actividad 5, el profesor comentó en el foro general que varios alumnos habían copiado 
la actividad (sin indicar los nombres) y explicó cómo se debe utilizar la información disponible y cómo citar las fuentes, también advirtió sobre las consecuencias de la copia o plagio en los trabajos (suspenso de la asignatura). Tras este mensaje, el profesor

\section{Discusión e implicaciones}

Algunos autores han destacado la importancia que tiene alinear las competencias que se definen para una asignatura con las actividades que realiza el estudiante (Guerrero Roldán et al., 2013). En este trabajo se ha realizado un estudio exploratorio que ha permitido, por un lado, identificar las competencias que se pueden desarrollar en un contexto formativo virtual y, por otro, analizar las posibles diferencias que hay entre las percepciones del profesor y del alumno con relación al grado en que se puede desarrollar una competencia a través de la realización de determinadas actividades. En este sentido, la percepción del alumno puede diferir bastante de la del profesor, por lo que podría ocurrir que las actividades propuestas por este último no sirvan para desarrollar ciertas competencias por parte del primero. De esta forma, nuestro análisis debería alertar al profesor sobre cuáles son las actividades que realmente funcionan y cuáles no en la generación de cada competencia.

Para ello, tras identificar las competencias genéricas y específicas que más se adaptan a la formación on-line, se han propuesto observó que los alumnos utilizaron y citaron varias fuentes de información para realizar la última actividad. Otro aspecto que puede tener efecto en la mejora del compromiso ético es el hecho de que el trabajo se realizó en grupo.

una serie de actividades que, en opinión del profesor, deberían ayudar a generar dichas competencias, en otras palabras, se han vinculado e interrelacionado competencias y actividades. Además y con la finalidad de conocer al grado de percepción del estudiante, se ha preguntado a los alumnos sobre el grado de mejora de sus competencias así como de si las actividades propuestas han contribuido al desarrollo de dichas competencias. De esta forma, al comparar la percepción del profesor con la del estudiante, deberíamos obtener información que nos permita reformular las actividades propuestas para trabajar cada competencia. En este sentido, resulta de especial interés que el profesor defina claramente qué entiende por cada competencia, es decir, puede que la percepción del profesor y del alumno sobre una determinada competencia difiera, por ejemplo, la creatividad o el liderazgo. Si el profesor no aclara al alumno qué entiende por estas competencias, puede que la valoración se esté llevando a cabo en términos diferentes. Sirva como ejemplo la competencia referida a la motivación por la calidad valorada de forma 
recurrente en casi todas las actividades por parte del alumno, percepción que no coincide con la valoración realizada por el profesor. Este hecho parece lógico, ya que lo contrario supondría un reconocimiento por parte del estudiante de que no está motivado a hacer un trabajo de calidad. En este sentido, lo que sí ha podido comprobarse es una mejora importante en la calidad de los trabajos a lo largo del cuatrimestre.

Los resultados muestran que el alumno percibe y entiende que las actividades realizadas le han permitido desarrollar en mayor o menor medida todas las competencias definidas en la guía docente. Sin embargo, si se reflexiona sobre este hecho, lo que se deduce es que el alumno toma como punto de partida la percepción que tiene sobre el grado en que tiene disponible una competencia para valorar la aportación o mejora de la misma con el desarrollo de una o varias actividades. Por ejemplo, en el caso de la capacidad para trabajar en grupo, aunque casi todas las actividades se han realizado de manera individual, los alumnos han valorado esta competencia por encima del 5 en todas las actividades.

Resulta especialmente destacable el resultado obtenido para la competencia compromiso ético en el trabajo. En términos estadísticos no se observan diferencias significativas en los valores medios antes y después de cursar la asignatura; sin embargo es interesante analizar con más detalle los datos. Antes de cursar la asignatura, trece alumnos valoraron su comportamiento ético entre 5 y 7 , quince consideraron que tenían un grado de 8, cuatro de 9 y cinco de 10 . Una vez cursada la asignatura, y teniendo en cuenta la situación que se planteó cuando se realizó la actividad 5 (varios alumnos copiaron la solución de una página de internet) y el feedback que dio el profesor ante este hecho, los alumnos que valoraron su ética con un 8 mantuvieron esta puntuación; mientras que todos los que consideraron que tenían un grado de 6 y dos de los que pusieron 7 , tras cursar la asignatura pasaron a puntuar esta competencia con un 9; y uno de los que la valoró inicialmente con 9 pasó a 10. Estos resultados son una muestra de la importancia que tiene que el alumno sea consciente de lo que hace, de cómo lo hace y de las consecuencias que puede tener un comportamiento no ético. Esta situación pone de manifiesto un problema que se está dando de forma recurrente en el panorama educativo: el plagio o copia en los trabajos. Para evitarlo se están desarrollando sistemas que permitan detectar esta mala práctica como el programa Turnitin (Matías-Pereda \& Lannelongue, 2013) y se está insistiendo en la necesidad de explicar a los alumnos en qué consiste el plagio y cuáles son las buenas prácticas de citación (Comas Forgas, Sureda Negre, \& Oliver Trobat, 2011).

Otro resultado destacable es que, cuando el alumno trabaja en grupo, percibe un mayor desarrollo de competencias, lo cual, curiosamente, coincide con la realización de la última actividad del curso. En este sentido, la percepción de haber adquirido un mayor número de competencias puede deberse 
tanto al tipo de actividad realizada en grupo como al momento en que se ha realizado. Otra implicación que puede desprenderse de esa situación es que resulta recomendable realizar una planificación temporal del tipo de actividad a realizar en función de las competencias que se quiera trabajar. En este caso, el docente debería plantearse si una actividad en grupo no habría sido más recomendable en un momento diferente del tiempo.

Por otra parte, los criterios de evaluación de cada actividad deben reflejar el grado de adquisición de cada competencia (Guerrero Roldán et al., 2013). En este trabajo no se ha analizado la forma de evaluar cada actividad a través de las competencias pero los resultados del mismo han planteado varios retos. Por una parte, tanto profesor como alumno entenderían y valorarían mejor $\mathrm{su}$ aprendizaje a través de las competencias si además de identificar qué competencias se trabajan con cada actividad, se incluyen los niveles que puede tener cada competencia (nivel mínimo y máximo) y hasta qué nivel se quiere trabajar. En este sentido, Guerrero Roldán et al. (2013) ofrecen un ejemplo para una asignatura (Lógica). Para cada competencia definen tres niveles e indican qué nivel se exige en esta asignatura y la forma en que cada competencia puede ser trabajada en varias actividades.

Los resultados obtenidos pueden permitir a los profesores plantear actividades y usar metodologías docentes más apropiadas para el desarrollo de las competencias; y al alumno desarrollarlas, entender su utilidad y conocer cuál es su aplicación práctica.

Finalmente, este estudio ofrece nueva evidencia empírica y puede servir de referencia para que los docentes analicen la adecuación de las competencias formuladas en sus guías docentes y, si fuera necesario, reformulen dichas competencias.

\section{Agradecimientos}

Este trabajo ha sido financiado por el Economía y Competitividad. Proyecto ECO2012-36775 del Ministerio de

\section{Referencias}

Abad Guerrero, I. M., \& Castillo Clavero, de ICE, 2795, 29-41.

A. M. (2004). Desarrollo de competencias directivas. Ajuste de la formación universitaria Acosta Lugo, W. (2004). Aproximación a la realidad empresarial. Boletín Económico a e-learning y el aprendizaje a distancia. 
Learning Review, 1. Recuperado el 24 de enero, 2014, de http://goo.gl/yG9QXW.

Aguaded Gómez, J. I., Tirado Morueta, R., \& Hernando Gómez, A. (2011). Campus virtuales en universidades andaluzas: tipologías de uso educativo, competencias do-centes y apoyo institucional. Teoría de la Educación: Revista Interuniversitaria, 23(1), 159-179.

ANECA (2005). Libro Blanco Título de Grado en Economía y Empresa. Madrid: ANECA.

Avargil, S., Herscovitz, O., \& Dori, Y. (2012). Teaching thinking skills in context based learning: Teachers challenges and assessment knowledge. Journal of Science Education and Technology, 21(2), 207-225. http://dx.doi. org/10.1007/s10956-011-9302-7

Betegón Sánchez, L., Fossas Olalla, M., Martínez Rodríguez E., \& Ramos González, M. M. (2010). Entornos virtuales como apoyo a la docencia universitaria presencial: utilidad de Moodle. Anuario Jurídico y Económico Escurialense, 43, 273-302.

Bustos Lambert, C., Arjona Fuentes, J. M., \& Celaya Pomposo, A. M. (2007). El desarrollo de competencias en Dirección Estratégica a través del aprendizaje cooperativo. Comunicación presentada en las V Jornadas de Investigación en Docencia Universitaria, Alicante, España. Camisón Zornoza, C., Boronat Navarro, M., Ariel.
Ceular Villamandos, N., Núñez Tabales, J. M., Serrano Muñoz, A., \& Caridad y Ocerin, J. M. (2012). El diseño de la asignatura de Dirección Estratégica en el nuevo Grado de Relaciones Laborales y Recursos Humanos en la Universidad de Córdoba. Revista d'Innovació Docent Universitària, 4, 41-48.

Comas Forgas, R., Sureda Negre, J., \& Oliver Trobat, M. (2011). Prácticas de citación y plagio académico en la elaboración textual del alumnado universitario. Revista Teoría de la Educación: Educación y Cultura en la Sociedad de la Información, 12(1), 359-385.

Duart, J. M. (2010). Nuevas brechas digitales en la educación superior. Revista de Universidad y Sociedad del Conocimiento, $7(1), 1-2$.

García Aretio, L. (1986). Educación superior a distancia. Análisis de su eficacia. Badajoz: UNED-Mérida.

García Aretio, L. (2001). Educación a distancia. De la teoría a la práctica. Barcelona: 
García Aretio, L., \& Ruiz Corbella, M. (2010). La eficacia en la educación a distancia: ¿Un problema resuelto?. Teoría de la Educación: Revista Interunivertaria, 22(1), 141-162.

García Manjón, J. V., \& Pérez López, M.

C. (2008). Espacio Europeo de Educación Superior, competencias profesionales y empleabilidad. Revista Iberoamericana de Educación, 46(9), 1-12.

García Martín, A. (2010). Manual de elaboración de guías docentes adaptadas al EEES. Murcia: Universidad Politécnica de Cartagena. Recuperado el 4 de febrero, 2014 de http://goo.gl/zSvwSH.

Gargallo Castel, A., \& Pérez Sanz, J. (2008). Metodologías activas en la dirección estratégica de la empresa. Implicación de los alumnos en el análisis estratégico de la Universidad. II Jornadas de Innovación Docente, Tecnologías de la Información y de la Comunicación e Investigación Educativa en la Universidad de Zaragoza.

González Díaz-Caneja, I., López García, A. M., \& Chasco Irigoyen, C. (2003). El e-learning en la universidad española. XVII Reunión Anual Asepelt-España, Almería, España.

González J., \& Wagenaar, R. (2003). Tuning educational structures in Europe. Bilbao: Universidad de Deusto.
Guerrero Serón, A. (1999). El enfoque de las competencias profesionales: Una solución conflictiva a la relación entre formación y empleo. Revista Complutense de Educación, 10(1), 335-360.

Guerrero-Roldán, A. E., Huertas, M. A., Mor, E., \& Rodríguez, M. E. (2013). Explicitando la interrelación entre las actividades de aprendizaje, el proceso de evaluación y la adquisición de competencias. RIED. Revista Iberoamericana de Educación a Distancia, 16, 127-146. http://dx.doi.org/10.5944/ ried.16.2.9909.

Laviña, J., \& Mengual, J. (2008) Libro blanco de la Universidad Digital 2010. Madrid: Editorial Ariel.

Martín Galán, B., \& Rodríguez Mateos, D. (2012). La evaluación de la formación uni-versitaria semipresencial y en línea en el contexto del EEES mediante el uso de los informes de actividad de la plataforma Moodle. RIED. Revista Iberoamericana de Educación a Distancia, 15(1), 159-178. http://dx.doi.org/10.5944/ried.1.15.782.

Matías-Pereda, J., \& Lannelongue, G. (2013). Técnicas de ayuda en el proceso de aprendizaje: El caso de los sistemas anticopia. Revista Teoría de la Educación: Educación y Cultura en la Sociedad de la Información, 14(1), 170-187. 
Mondéjar, J. A., Mondéjar, J., \& Vargas, M. Oliveros Martín-Varés, L. (2006). (2007). Docencia virtual en universidades Identificación decompetencias: Una estrategia presenciales: Experiencia en la Universidad para la formación en el Espacio Europeo de de Castilla-La Mancha, RIED. Revista Educación Superior. Revista Complutense de Iberoamericana de Educación a Distancia, Educación, 17, 101-118. 10(2), 207-228. http://dx.doi.org/10.5944/ ried.2.10.1000.

Pavón Rabasco, F., \& Casanova Correa, J. (2007). Experiencias docentes apoyadas en Montoro-Sánchez, M. A., Mora-Valentín, E. aulas virtuales, RIED. Revista Iberoamericana M., \& Ortiz-de-Urbina-Criado, M. (2012). de Educación a Distancia, 10(2), 149-163. Análisis de las competencias adquiridas en los http://dx.doi.org/10.5944/ried.2.10.997. estudios de Dirección de Empresas y su grado de aplicación en las prácticas en empresas. Pereda, S., Berrocal, F., \& López, M. Revista Complutense de Educación, 23, 241- (2002). Gestión de recursos humanos por 263. competencias y gestión del conocimiento. http://dx.doi.org/10.5209/rev_rced.2012. Dirección y Organización, 28, 43-54. v23.n1.39112

\section{Notas}

\footnotetext{
${ }^{1}$ Un análisis más detallado de estas competencias puede consultarse en Montoro-Sánchez, Mora-Valentín, \& Ortiz-de-Urbina-Criado (2012).

${ }^{2}$ Otros trabajos que se centran en la asignatura de Dirección Estratégica son Bustos Lambert et al. (2007), Camisón Zornoza, Boronat

Navarro, \& Villar López (2007), Gargallo Castel \& Pérez Sanz, (2008) y Ceular Villamandos et al. (2012).

${ }^{3}$ Las tablas de frecuencias quedan a disposición de los lectores bajo petición a los autores.
} 